\title{
Shell Architecture Strongly Influences the Glass Transition, Surface Mobility, and Elasticity of Polymer Core-Shell Nanoparticles
}

\author{
Eunsoo Kang, ${ }^{\dagger}$ Bartlomiej Graczykowski, ${ }^{\dagger}{ }^{\dagger}$ Ulrich Jonas, ${ }^{\S}$ (๑ Dane Christie, " Laura A. G. Gray, \\ Daniele Cangialosi, ${ }^{\#, \nabla}$ Rodney D. Priestley, ${ }^{\|, \perp}{ }^{(0)}$ and George Fytas ${ }^{*} \dagger$ \\ ${ }^{\dagger}$ Max Planck Institute for Polymer Research, Ackermannweg 10, 55128 Mainz, Germany \\ ${ }^{\ddagger}$ Faculty of Physics, Adam Mickiewicz University, Umultowska 85, 61614 Poznan, Poland \\ ${ }^{\S}$ Department of Chemistry and Biology, University of Siegen, Adolf-Reichwein-Strasse 2, 57076 Siegen, Germany \\ ${ }^{\|}$Department of Chemical and Biological Engineering and ${ }^{\perp}$ Princeton Institute for the Science and Technology of Materials, \\ Princeton University, Princeton, New Jersey 08544, United States \\ ${ }^{\#}$ Centro de Física de Materiales (CSIC-UPV/EHU), Paseo Manuel de Lardizabal 5, 20018 San Sebastián, Spain \\ ${ }^{\nabla}$ Donostia International Physics Center, Paseo Manuel de Lardizabal 4, 20018 San Sebastián, Spain
}

Supporting Information

ABSTRACT: Despite the growing application of nanostructured polymeric materials, there still remains a large gap in our understanding of polymer mechanics and thermal stability under confinement and near polymer-polymer interfaces. In particular, the knowledge of polymer nanoparticle thermal stability and mechanics is of great importance for their application in drug delivery, phononics, and photonics. Here, we quantified the effects of a polymer shell layer on the modulus and glass-transition temperature $\left(T_{\mathrm{g}}\right)$ of polymer coreshell nanoparticles via Brillouin light spectroscopy and modulated differential scanning calorimetry, respectively. Nanoparticles consisting of a polystyrene (PS) core and shell layers of poly $(n$-butyl
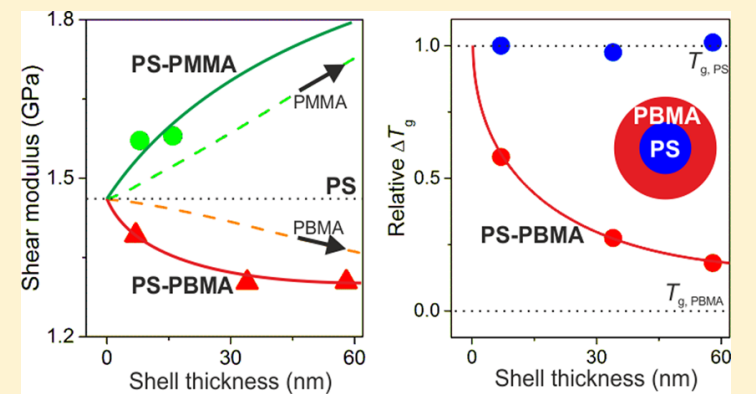
methacrylate) (PBMA) were characterized as model systems. We found that the high $T_{\mathrm{g}}$ of the PS core was largely unaffected by the presence of an outer polymer shell, whereas the lower $T_{\mathrm{g}}$ of the PBMA shell layer decreased with increasing PBMA thickness. The surface mobility was revealed at a temperature about $15 \mathrm{~K}$ lower than the $T_{\mathrm{g}}$ of the PBMA shell layer. Overall, the modulus of the core-shell nanoparticles decreased with increasing PBMA shell layer thickness. These results suggest that the nanoparticle modulus and $T_{\mathrm{g}}$ can be tuned independently through the control of nanoparticle composition and architecture.

\section{INTRODUCTION}

Nanoscaled polymeric materials are increasingly used in advanced technologies, for example, as separation membranes, ${ }^{1,2}$ drug-delivery vehicles, ${ }^{3}$ reinforcing modifiers, ${ }^{4}$ and photonic ${ }^{5}$ and phononic crystals. ${ }^{6}$ These technologies utilize one-dimensional (e.g., thin films) and three-dimensional (e.g., nanoparticles (NPs)) confinement to modify polymer properties. When the length scale of a polymeric system is reduced to the nanoscale, polymer materials show deviations in physical properties $^{7-9}$ such as viscosity, ${ }^{10-12}$ elasticity, ${ }^{13,14}$ and glasstransition temperature $\left(T_{\mathrm{g}}\right)$ from the bulk. ${ }^{15-23}$ Although the mechanism behind these deviations is of much debate, interfacial and surface effects dominate confined polymer properties due to the large ratio of surface area to volume.

The influence of polymer-polymer interfaces on the $T_{\mathrm{g}}$ of confined polymers remains an open question. Polymer $T_{\mathrm{g}}$ near the polymer-polymer interface has been observed in stacked multilayer films ${ }^{24-28}$ and phase-separated block copolymers. ${ }^{29,30}$ The interfacial width, contrast between bulk $T_{\mathrm{g}}$ values, chain connectivity, and relative Debye-Waller factors may all play a role in determining the change in $T_{\mathrm{g}}$ across a polymer-polymer interface. ${ }^{30,31}$ Less well-studied is the influence of polymer-polymer interfaces on the mechanical properties of confined polymers. ${ }^{13,32-36}$ Various techniques including buckling, ${ }^{13}$ nanobubble inflation, ${ }^{32}$ and nanoindentation ${ }^{34,37}$ have been used to measure the Young modulus or compliance of polymer thin films both above and below the $T_{\mathrm{g}}$. Below the $T_{\mathrm{g}}$, both a substantial reduction $^{13,33}$ and an increase ${ }^{37}$ in the Young modulus, depending on the nature of the underlying substrate, have been measured for films with a thickness less than $\sim 40 \mathrm{~nm}$. In rubbery films, above the $T_{g}$, stiffening has been observed under nanoscale confinement. ${ }^{32}$ Although these experiments have been conducted in a variety of geometries including freestanding films, films supported atop elastomers, and films with and without a topcoat polymer layer supported atop silicon, a thorough investigation of the mechanical properties near polymer-polymer interfaces of confined systems has not yet

Received: April 15, 2019

Revised: June 14, 2019

Published: July 11, 2019 
Table 1. Dimensions of Shell Architecture of PS-Based Core-Shell Nanoparticles

$\begin{array}{llllll}\text { samples } & \text { PS-PS } & \text { PS-PBMA } & \text { PS-PBMA }_{34} & \text { PS-PBMA }_{58} & \text { PS-PMMA }_{8} \\ \text { core diameter }^{a}(\mathrm{~nm}) & 222 \pm 7 & 220 \pm 7 & 222 \pm 7 & 201 \pm 9 & 220 \pm 7 \\ \text { NP diameter }^{a}(\mathrm{~nm}) & 256 \pm 6 & 234 \pm 9 & 290 \pm 11 & 317 \pm 12 & 236 \pm 7 \\ \text { shell thickness } h(\mathrm{~nm}) & 18 \pm 3 & 7 \pm 5 & 34 \pm 5 & 58 \pm 6 & 8 \pm 3 \\ \text { shell vol. fraction } & 0.35 \pm 0.1 & 0.17 \pm 0.07 & 0.55 \pm 0.1 & 0.75 \pm 0.08 & 0.19 \pm 0.1\end{array}$

${ }^{a}$ Particle size was measured by SEM images.

been undertaken. To understand the effect of polymer interfaces on the modulus and $T_{g}$, we recently introduced polystyrene (PS) core-shell NPs with varying ultrathin shells via seeded surfactant-free polymerization and layer-by-layer adsorption. ${ }^{38}$ We found a significant change in the elastic modulus and thermal behavior of NPs by the introduction of ultrathin shell layers with different chemical compositions via Brillouin light-scattering spectroscopy (BLS). ${ }^{38}$

Recently, focus has been placed on studying deviations in the glass transition of nanoparticles. ${ }^{38-41}$ Spherical geometries have received less attention partially due to the limited number of experimental techniques available to measure the material properties of nanoparticles. Zhang et al. ${ }^{40}$ first measured a decrease in the $T_{\mathrm{g}}(\sim 50 \mathrm{~K})$ for $\sim 90 \mathrm{~nm}$ PS NPs prepared by surfactant-free emulsion polymerization. When capped with a silica shell, the $T_{\mathrm{g}}$ confinement effect was absent due to the removal of the free surface. ${ }^{40}$ Feng et al. ${ }^{41}$ modified PS NPs with different types of surfactants and discovered a strong surfactant effect on $T_{\mathrm{g}}$ deviations for NPs less than $200 \mathrm{~nm}$ in diameter, thus emphasizing the importance of the NP surface in determining confinement effects. Furthermore, recent measurements of molecular mobility in $\mathrm{NPs}^{42,43}$ have shown a decoupling between the decrease in the NP $T_{\mathrm{g}}$ with decreasing diameter and no change in the segmental mobility as measured by dielectric relaxation spectroscopy ${ }^{42}$ and fastscanning calorimetry. ${ }^{43}$

Here, we examine PS-core NPs with a low- $T_{\mathrm{g}}$ poly(butyl methacrylate) (PBMA) shell layer of varying thickness to understand the effects of the outermost surface and core-shell interface on the thermomechanical properties of the shell. The PS core was synthesized by surfactant-free polymerization. Seeded surfactant-free polymerization was adapted to produce the PS-PBMA and PS-poly(methyl methacrylate) (PMMA) core-shell NPs. The synthesis of core-shell NPs is elaborated in the Supporting Information. BLS with a high-resolution tandem Fabry-Perot interferometer was used to measure the elastic properties, particle surface-softening temperature $\left(T_{\mathrm{s}}\right)$, and $T_{\mathrm{g}}$. Modulated differential scanning calorimetry (mDSC) was used to measure the $T_{\mathrm{g}}$ of the NPs. To understand the influence of the shell layer on the thermomechanical properties via $\mathrm{BLS}$ and $\mathrm{mDSC}$, we have also investigated the thermal behavior of contiguous films prepared by annealing NPs.

\section{EXPERIMENTAL SECTION}

Preparation of Polymer Core-Shell Nanoparticles (NPs). The PS core was prepared by surfactant-free polymerization ${ }^{44}$ as a seed for PS-based core-shell NPs (PS-PS, PS-PBMA, PS$\mathrm{PBMA}_{34}, \mathrm{PS}-\mathrm{PBMA}_{58}, \mathrm{PS}-\mathrm{PMMA}_{8}$, and PS-PMMA 16 ). For the PS core, styrene and acrylic acid were adopted as a monomer and a comonomer, respectively. Acrylic acid was added to stabilize the particles. For the core-shell structure, each shell (PBMA and PMMA) was polymerized atop the PS core by a two-step surfactantfree polymerization. Different quantities of the monomer were used to change the shell thickness. As the shell was grown atop the core particles, some surface activation enabling partial chain grafting to the seed particle surface is conceivable. ${ }^{45}$ The growing chain of shell polymers in aqueous media diffuses to the surface of the core and forms a coating structure shell. ${ }^{46}$ After the polymerization, the unreacted monomer and impurities were removed by a mixture of high-purity ethanol and Milli-Q water. The particle dimensional information is listed in Table 1 , and the detailed NP preparation procedure is in the Supporting Information.

Brillouin Light-Scattering Spectroscopy (BLS). BLS experiments were performed for polymer nanoparticle colloids and annealed films in transmission geometry by means of a six-pass tandem FabryPerot interferometer. The scattering (acoustic) wave vector $\mathbf{q}$ is defined as $\mathbf{q}=\mathbf{k}_{\mathrm{s}}-\mathbf{k}_{\mathrm{i}}$, where $\mathbf{k}_{\mathrm{s}}$ and $\mathbf{k}_{\mathrm{i}}$ are the wave vectors of scattered and incident light, respectively. In transmission geometry, $\mathbf{q}$ is parallel to the sample plane and is of the magnitude $q=(4 \pi / \lambda)$ $\sin (\theta / 2)$, where $\theta$ is the angle between the incident light and scattered light and $\lambda$ is the wavelength of the incident laser light. In our study, we used a laser operating at $\lambda=532 \mathrm{~nm}$, fixed $\theta=90^{\circ}(q=0.0167$ $\mathrm{nm}^{-1}$ ), and vertical-vertical (vv) polarization of incident and scattered light, respectively. The temperature was diversified with a home-built temperature-scanning apparatus to observe the thermal behavior of nanoparticles.

Modulated Differential Scanning Calorimetry (mDSC). To measure the glass-transition temperature of core-shell nanoparticles, mDSC, TA Instruments Q2000, was used. The samples were heated at $20 \mathrm{~K} / \mathrm{min}$ with $0.2 \mathrm{~K}$ per $20 \mathrm{~s}$ modulation. Before the measurement, nanoparticle suspensions were dried under vacuum at room temperature or annealed at $413 \mathrm{~K}$ overnight into a film.

\section{RESULTS AND DISCUSSION}

Nanoparticle Shear Elastic Modulus. Figure 1 shows the anti-Stokes side of the BLS spectra of four different closepacked clusters of core-shell NPs, PS-PS, PS-PMMA ${ }_{8}$, PS$\mathrm{PBMA}_{7}$, and $\mathrm{PS}-\mathrm{PBMA}_{34}$, at $294 \mathrm{~K}$ with the particle size information listed in Table 1. For all core-shell NPs, the diameter was obtained from the SEM images, averaging the size of 100 randomly chosen particles. The identification number within the nomenclature represents the thickness of the shell in nanometers and a change in the shell volume fraction. In the quasi-core-shell PS-PS NP, the thin PS shell layer, which has the same chemistry as the PS core, shows negligible effects on the particle elasticity ${ }^{38}$ and hence is used as a reference. To compare the spectral features of systems differing both in structure and size, we account for the latter by scaling the particle vibration frequencies by the NP diameter (d). The BLS spectra were then plotted against the particle diameter normalized frequency $(f \cdot d)$ as shown in Figure 1a. In principle, BLS probes the spheroidal mechanical eigenmodes of NPs. The eigenfrequencies of these modes are labeled $f(n, l)$, where $n$ and $l$ are integers defining the radial and angular dependence of the displacement, respectively. ${ }^{47}$ To determine the thermomechanical properties of NPs, we focused on the spectral position and line shape of the $(1,2)$ mode. Here, 1 and 2 stand for the radial and angular vibrations of spheres, respectively. ${ }^{48}$ As depicted in Figure 1, in each case, the $(1,2)$ modes correspond to Lorentzian doublets (solid gray lines) peaked at $f_{1}$ and $f_{2}$. The split and blue shift of the $f(1,2)$ with respect to the isolated NPs is the result of attractive 




b



Figure 1. (a) Anti-Stokes side of the Brillouin light-scattering (BLS) vibrational spectra of the shell architecture of PS-PS, PS-PMMA PS $-\mathrm{PBMA}_{7}$, and PS-PBMA 34 vs reduced frequency, $f \cdot d$, with $d$ being the particle diameter. The frequencies $f_{1}, f_{2}$, denote the spectral split of the $(1,2)$ mode and the vertical dotted lines indicate $f_{1}, f_{2}$, and $f(1,3)$ of the PS-PS NPs. (b) BLS power spectra, $I \cdot f^{2}$, vs frequency, $f$, at $294 \mathrm{~K}$. The arrows indicate the interaction-induced $(1,1)$ branch.

interactions between NPs in a colloidal cluster. ${ }^{47-53}$ The $(1,2)$ peak position clearly shifts depending on the chemical composition and thickness of the shell as indicated by the two dashed vertical lines in Figure 1a. For example, the $f(1,2)$ of PS-PBMA undergo a red shift compared to the quasi-coreshell PS-PS NPs. The blue (red) shift in the spectra implies a stiffening (softening) of the NP modulus. Interestingly, the enhanced modulus of the PS-PMMA core-shell NPs is independent of the PMMA shell layer thickness (see Figure S1). For the PS-PBMA NPs, the red shift of $(1,2)$ increases with the thickness of the PBMA shell layer from PS-PBMA, to PS-PBMA 58 (Figure S1). This trend reflects a reduction in the NP modulus as a function of the PBMA shell layer thickness, as discussed in detail below.

To account for the thermal phonon population and better reveal the low-frequency side of the BLS spectra near the elastic Rayleigh peak, Figure $1 \mathrm{~b}$ displays the power spectra $I \cdot f^{2}$. A clear low-frequency peak (arrows) appears in the power spectrum. This $(1,1)$ mode $^{47}$ is related to longitudinal phonons in the cluster of the NPs induced by their mutual adhesion, and its frequency $f(1,1)$ can be described by the JohnsonKendall-Roberts model. ${ }^{54}$ The precise $f(1,1)$ position is defined as the cutoff frequency in the right tail where the intensity is $20 \%$ of the $f(1,1)$ maximum intensity in the power spectrum. When this peak position is plotted against diameter, it follows the scaling $f(1,1) \sim d^{-7 / 6}$ as shown in Figure S2. Although all core-shell NPs follow the scaling because of a similar adhesion energy, ${ }^{52}$ PS- $-\mathrm{PBMA}_{58}$ data deviated from the solid line, suggesting a higher adhesion energy.

We have recently reported that $f_{\mathrm{L}}(1,2)=2 f_{1}-f_{2}$ can account for the effect of interparticle interactions, providing a good approximation of the frequency $f(1,2)$ of the single $(1,2)$ mode of individual NPs $\left(f_{1}=f_{2}\right)$. ${ }^{52}$ To estimate the shear modulus (G) of NPs, the equation

$C_{\mathrm{t}}=f_{\mathrm{L}}(1,2) \cdot d / A=\sqrt{G / \rho}$ was applied. ${ }^{55}$ Here, $C_{\mathrm{t}}$ is the effective transverse sound velocity of NPs, $d$ is the diameter, and $\rho$ is density of particles. The value of the Lamb constant, $A$, depends on the Poisson ratio (Figure S3), and for the present case, $A=0.84$ for PS spheres was used. Figure 2

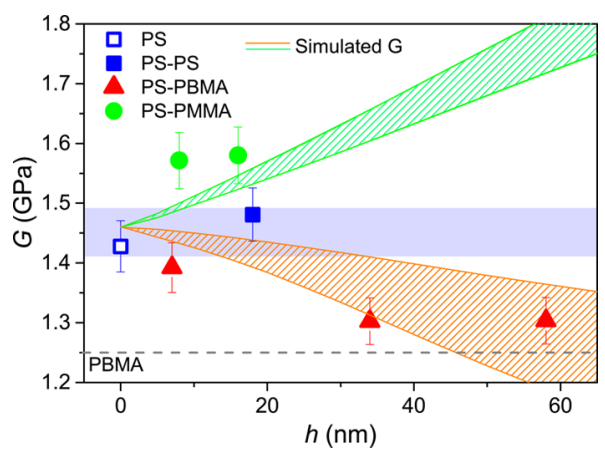

Figure 2. Shear modulus, G, of PS and core-shell architecture, PS$\mathrm{PS}$, the PS-PBMA group (PS-PBMA, $\mathrm{PS}-\mathrm{PBMA}_{34}$, and PS$\mathrm{PBMA}_{58}$ ), and the PS-PMMA group (PS-PMMA 8 and PSPMMA $_{16}$ ), NPs plotted against the shell thickness, $h$. The dashed straight line indicates the shear modulus for the bulk PBMA, whereas the open square and solid circles refer to the shear modulus of the precursor PS and PS-PMMA core-shell nanoparticles. ${ }^{38}$ The red triangles refer to the shear modulus of PS-PBMA core-shell NPs. The shaded area indicates the maximum error for the modulus of PS. The red and green solid lines indicate the calculated shear modulus for PS-PBMA and PS-PMMA core-shell NPs at a constant PS core diameter $(d=220 \mathrm{~nm})$ using bulk moduli values for the PS core and two different values for the PMMA $\left(C_{\mathrm{t}}=1500\right.$ and $\left.1400 \mathrm{~m} / \mathrm{s}\right)$ and PBMA $\left(C_{\mathrm{t}}=900\right.$ and $\left.1050 \mathrm{~m} / \mathrm{s}\right)$ shells. A similar plot for $G$ but as function of the shell volume fraction is shown in Figure S4 in the Supporting Information.

depicts the $G$ vs shell thickness plot for the studied NPs (the plot for $G$ vs shell volume fraction is shown Figure $S 4$ ); $C_{\mathrm{t}}$ and $G$ values for the NPs are listed in Table S1. Note that given the high frequency of the NP vibrations, $G$ refers to the elastic (frequency-independent) modulus. As the thickness of the soft PBMA shell increases, $C_{t}$ and $G$ decrease. In Figure 2, the estimated shear modulus of the NPs is plotted against shell thickness. Based on previous studies, ${ }^{38,52}$ the particle size does not affect the shear modulus, but the chemical composition plays a more important role in determining the NP shear modulus in homogeneous NPs. PS and the quasi-PS-PS core-shell NPs have a similar shear modulus in spite of their different particle sizes and polymerization processes. The shellforming process has no discernible effect on the shear modulus, but the chemical composition of the shell can impact the shear modulus of NPs. ${ }^{38}$ PS NPs with a highmodulus PMMA shell manifest about a 10\% higher modulus than their precursor PS core even with the addition of a thin $(8$ $\mathrm{nm}$ ), harder shell layer. The hardening trend is also observed for the PS-PMMA 16 NPs, but it is mainly due to the increase of density as the NPs' $C_{\mathrm{t}}$ remains virtually constant; there is no significant blue shift for PS-PMMA 16 (see Figure S1). Compared to PS-PS NPs, a lower shear modulus was revealed for PS-PBMA NPs. The NP modulus decreased with increasing PBMA thickness, reaching a value $\sim 12 \%$ lower than that of PS-PS NPs for a $58 \mathrm{~nm}$ PBMA shell (see Figure 2 ). The enhancement of the modulus in the case of the PSPMMA NP corroborates the notion that a thin shell layer of an 
elastically different material, atop the PS core, can significantly modify the core-shell NP elasticity.

The finite element method (FEM) was used to calculate the shear modulus of the PS-PMMA and PS-PBMA NPs assuming no interactions among NPs and perfect bonding between the core and shell interface and bulk elastic properties of each component (see Figure S5). In this case, the $(1,2)$ peak is single in contrast to the interaction-induced double-spectral shape (see Figures 1 and S1, S5). ${ }^{52}$ Using $C_{\mathrm{t}}=1400 \mathrm{~m} / \mathrm{s}$ for $\mathrm{PMMA}^{56}$ and $C_{\mathrm{t}}=1050 \mathrm{~m} / \mathrm{s}$ PBMA, $G$ is presented by the lower green and upper red lines in Figure 2. For PBMA, $C_{t}$ was obtained from the experimental longitudinal value $C_{\mathrm{L}}=2200$ $\mathrm{m} / \mathrm{s}$ and using the value 0.35 for the Poisson ratio. The agreement is only qualitative, since the calculated $G$ overestimates the experimental value of $G$ of PS-PBMA (upper red line, $C_{\mathrm{t}}=1050 \mathrm{~m} / \mathrm{s}$ ) and underestimates the $G$ value for the two PS-PMMA NPs (lower green line, $C_{\mathrm{t}}=1400 \mathrm{~m} / \mathrm{s}$ ). To obtain quantitative agreement between FEM calculations and the experiment, the transverse sound velocity $C_{\mathrm{t}}$ for the PBMA shell should deviate from its bulk value. The lower red line in Figure 2 corresponds to $C_{t}=900 \mathrm{~m} / \mathrm{s}$, being about $15 \%$ lower than that in the bulk PBMA. The deviation between the assumed PBMA shell and PBMA bulk $C_{\mathrm{t}}$ values decreases with increasing PBMA shell thickness as indicated by the deviation of the experimental $G$ values from the two red lines. For a thinner PBMA shell, PS-PBMA $15 \%$ lower than that in the bulk PBMA $(1050 \mathrm{~m} / \mathrm{s})$, whereas for PS-PBMA ${ }_{58}$, its elastic $G$ can be captured with the bulk PBMA $C_{\mathrm{t}}$. In analogy, for the PS-PMMA NPs, a higher $C_{t}$ $(=1500 \mathrm{~m} / \mathrm{s})$ than for the bulk PMMA is required as indicated by the upper green line.

Although for colloids there are no other experiments on the elastic modulus, the impact of confinement on the Young modulus of polymer thin films has been investigated by buckling instability techniques, ${ }^{13,33,57}$ atomic force microscopy (AFM), 23,58,59 and a nanobubble inflation method. ${ }^{32} \mathrm{~A}$ reduction in the glassy modulus has been reported for thin $(<40 \mathrm{~nm})$ PS films atop elastic poly(dimethylsiloxane) substrates and has been assigned to a free surface layer with a reduced modulus. ${ }^{33,57}$ An enhancement of the glassy modulus was reported near interfaces when thin polymer films were supported on hard substrates (e.g., silicon, glass). ${ }^{23,37,58,59}$ Quasi-static finite-element simulations ${ }^{59}$ of the AFM experiment assuming elastic properties of the substrate and the glassy polymer (PMMA) film indicate stiffening near the rigid substrate. Very recent molecular dynamics simulations ${ }^{60}$ have further suggested that the stiffness gradient length scale increases with temperature from below to above the polymer glass transition. Both simulations are based on several elastic and energetic parameters for the polymer interactions with the substrate and the indenter. Hence, the determining factor for either reduction or enhancement of the modulus for film-substrate systems is still unclear. Another system of polymer nanofibers such as PS and Nylon-11 shows a dramatic elastic modulus increase as the diameter drops below $200 \mathrm{~nm} .{ }^{61,62}$ Very recently, the enhanced elasticity in films of polymer-tethered nanoparticles at a low grafting density was attributed to strong polymer-polymer interactions compared to densely tethered NPs with short chains. ${ }^{63}$ For the spherical confinement of Figure 2, the NP shear modulus can be rationalized assuming hardening for the PMMA and softening for the PBMA shell atop the same PS core.
Nanoparticle Surface Mobility and Glass Transition. The sensitive dependence of the $(1,2)$ branch on the NP adhesion was utilized to directly probe the NP surface mobility and identify $T_{s}$ as the temperature at which a crossover from the red to blue frequency shift was observed. ${ }^{52}$ It originates from the activation of the outermost surface mobility of NPs. ${ }^{38,52}$ At $T_{s}$, the surface of the polymer particles undergoes a glass-to-rubber crossover. At temperatures above $T_{s}$, interparticle interactions become activated and thereby increase the contact area between NPs. ${ }^{52}$ The resulting reinforced adhesion force is at the origin of the $f(1,2)$ blue shift above the $T_{s}$. The presence of a mobile surface layer was first reported by the whispering gallery mode light localization, which revealed a negative optical birefringence. ${ }^{64}$ The frequencies $f_{1}$ and $f_{2}$ of the $(1,2)$ branch obtained by the representation of the $(1,2)$ peak in BLS spectra by a double Lorentzian line shape are shown in Figure $3 \mathrm{a}$ for the PS-PS,
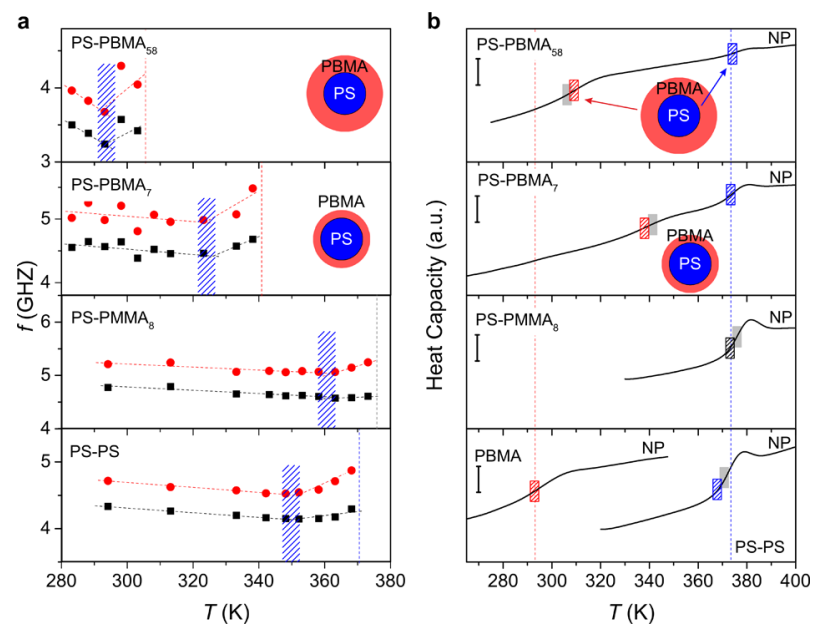

Figure 3. (a) Temperature dependence of the frequencies $f_{1}$ and $f_{2}$ for the $(1,2)$ branch in PS-PS, PS-PMMA ${ }_{8}, \mathrm{PS}-\mathrm{PBMA}_{7}$, and PS$\mathrm{PBMA}_{58}$ NPs. Vertical hatched squares indicate the softening transition temperature, $T_{s}$. The highest temperature at which vibration BLS is still resolved estimates the low glass-transition temperature, $T_{g,}$, of the NPs. $T_{\mathrm{g}}$ is marked with vertical dashed lines. (b) mDSC traces of dried powder for the indicated NPs. Shaded and hatched areas indicate, respectively, the glass-transition temperatures from the BLS and $\mathrm{mDSC}$ experiments. Red and blue hatched areas denote the low $T_{\mathrm{g}}\left(T_{\mathrm{g}, \mathrm{l}}\right)$ and high $T_{\mathrm{g}}\left(T_{\mathrm{g}, \mathrm{h}}\right)$, respectively.

PS-PMMA 8 , PS-PBMA , and PS-PBMA 58 NPs as a function of temperature (see Figure $S 6$ ). As temperature increases, the first crossover at $T_{\mathrm{s}}$ is indicated by vertical hatched squares. $T_{\mathrm{s}}$ is also resolved in the temperature dependence of the frequency $f(1,1)$ of the interaction-induced mode (see Figure S7). With further increase in temperature, the disappearance of the eigenmodes occurs at $T_{\mathrm{g}}$ where the polymer NPs transform into a contiguous film supporting a single acoustic phonon. This crossover from the particle-like vibration spectrum to a homogeneous BLS spectrum is shown in the heat maps of Figure S8. Such maps depict the frequencies (white circles) of the main peaks in the BLS spectrum at each recorded temperature. For the PS-PBMA NPs, no spectral peak was evident in the BLS spectrum recorded in the temperature (dark) region $\mathrm{B}$, as the coalescence of the PBMA shell rendered the resolution of the NPs' vibrations impossible. When the sample temperature exceeded this dark region, the sample transforms into a contiguous film and the BLS 
spectrum displayed only the longitudinal acoustic peak. In the case of PS-PBMA NPs, the incompatibility of the two components preserves the PS cores from merging within the annealed PBMA matrix, as indicated by the SEM images (see Figure S9). For PS-PS NPs, both $T_{\mathrm{s}}(=352 \mathrm{~K})$ and $T_{\mathrm{g}}(=371$ $\mathrm{K})$ assume the same values for the PS NPs in our previous work. $^{38}$ This indicates that the performed polymerization process, necessary for the formation of the shell, does not influence $T_{\mathrm{s}}$ or $T_{\mathrm{g}}$.

A thin $(8 \mathrm{~nm})$ PMMA shell atop PS NPs causes an increase of both $T_{\mathrm{s}}(\sim 8 \mathrm{~K})$ and $T_{\mathrm{g}}(\sim 5 \mathrm{~K})$ compared to those of PSPS NPs. As in the case of $G$, both transition temperatures are robust upon further increase of the PMMA thickness $(16 \mathrm{~nm})$ as the $T_{\mathrm{g}}$ has already reached the value for bulk PMMA. From the two characteristic temperatures of the NPs, only a single $T_{\mathrm{g}}$ can be measured by DSC as shown in Figure $3 \mathrm{~b}$. This $T_{\mathrm{g}}$ assumes the same value in both PS-PMMA ${ }_{8,16}$ NPs in agreement with the BLS, and it is indistinguishable, within experimental error, from the glass-transition temperature of PS-PS. For PS on PMMA bilayer films ${ }^{27}$ experiencing hard confinement next to PMMA (higher $T_{\mathrm{g}}$ than PS), the $T_{\mathrm{g}}$ in the PS domain deviated from the bulk $T_{\mathrm{g}}$ of PS below about 100 $\mathrm{nm}$ away from the PS/PMMA interface. A local $T_{\mathrm{g}}$ elevation of about $7 \mathrm{~K}$ was reported $25 \mathrm{~nm}$ from the PMMA layer, but no data exist for PMMA on the PS film geometry. The relatively small difference $(\sim 15 \mathrm{~K})$ in the bulk $T_{\mathrm{g}}$ between PS and PMMA limits the resolution of possible dynamic asymmetries in the PS core vs the PMMA shell. For a comparison, $\Delta T_{\mathrm{g}}=$ $T_{\mathrm{g}, \mathrm{PS}}-T_{\mathrm{g}, \mathrm{PBMA}} \approx 80 \mathrm{~K}$ between the PS and PBMA components.

For PS-PBMA NPs, the large dynamic asymmetry between the core and shell revealed (i) a strong dependence of both $T_{\mathrm{s}}$ and $T_{\mathrm{g}, 1}$ (see Figures 3a and S6a) and (ii) low and high glasstransition temperatures $\left(T_{\mathrm{g}, \mathrm{l}}, T_{\mathrm{g}, \mathrm{h}}\right)$ (see Figure $3 \mathrm{~b}$ ) on the PBMA thickness as in the case of $G$ (see Figure 2), but (iii) a robust value for $T_{\mathrm{g}, \mathrm{h}}=T_{\mathrm{g}, \mathrm{Ps}}$. BLS can access only $T_{\mathrm{s}}$ and $T_{\mathrm{g}, \mathrm{l}}$ due to the disappearance of the NP vibration at $T=T_{\mathrm{g}, 1}<T_{\mathrm{g}, \mathrm{PS}}$ (see Figures $3 \mathrm{a}$ and S6), whereas DSC probes both glass transitions (see Figure $3 b$ ). These characteristic temperatures for all systems are compiled in Table S2. The glass-transition enhancement and the relation between $T_{\mathrm{s}}$ and $T_{\mathrm{g}, 1}$ as a function of the proximity to the PS surface is illustrated in the lower and upper panels of Figure 4. To compare data of systems with different geometries, we plot normalized enhancements, $\Delta T_{\mathrm{g}}=$ $\left(T_{\mathrm{g}}(h)-T_{\mathrm{g}, \mathrm{PBMA}}\right) /\left(T_{\mathrm{g}, \mathrm{PS}}-T_{\mathrm{g}, \mathrm{PBMA}}\right)$, where $T_{\mathrm{g}}(h)$ is either $T_{\mathrm{g}, \mathrm{I}}$ (filled red circles) or high $T_{\mathrm{g}}(h)=T_{\mathrm{g}, \mathrm{h}}$ (blue solid circles). To appreciate the actual $T_{\mathrm{g}} \mathrm{s}$ changes, we have replotted Figure 4 as $T_{\mathrm{g}}$ vs $h$ in Figure S10.

The robustness of the PS $T_{\mathrm{g}}$ (solid blue circles in Figure $4 \mathrm{~b}$ ) is an unexpected result in view of the flat PBMA/PS bilayer films. For the latter, the glass-transition temperature was determined by fluorescence intensity measurements of pyrenelabeled layers (PS or PBMA) inserted at different distances, $h$, (dashed line in the insets in Figure $4 \mathrm{~b}$ ) from the PS/PBMA interface (solid line in the upper bilayer model). ${ }^{26}$ The $T_{\mathrm{g}}$ in the PS layer softens with proximity to the PBMA interface as indicated in Figure $4 \mathrm{~b}$ (blue open triangles) and reaches the bulk PS value at about $200 \mathrm{~nm}$ from the interface. Alternatively, the $T_{\mathrm{g}}$ in the PBMA layer is enhanced with increasing distance from the interface, but the dependence is weaker than that for the PS layer (open red squares). For the spherical geometry, the enhancement of the $T_{\mathrm{g}, 1}$ in the PBMA shell is stronger than that in the corresponding bilayer film

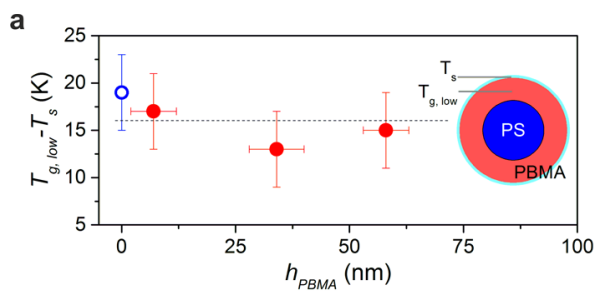

b



Figure 4. (a) Difference between the low glass-transition and the softening transition temperatures for the PS-PS and three PS-PBMA NPs depending on the shell thickness, $h$. (b) Normalized glasstransition temperature contrast, $\Delta T_{\mathrm{g}}=\left(T_{\mathrm{g}}(h)-T_{\mathrm{g}, \mathrm{PBMA}}\right) /\left(T_{\mathrm{g}, \mathrm{PS}}-\right.$ $\left.T_{\mathrm{g}, \text { PBMA }}\right)$, for the low $\left(T_{\mathrm{g}}(h)=T_{\mathrm{g}, \mathrm{t}}\right.$ filled red circles $)$ and high $\left(T_{\mathrm{g}}(h)\right.$ $=T_{\mathrm{g}, \mathrm{h}}$, blue solid circles) glass transitions in PS-PBMA as a function of the shell thickness. Note that $T_{\mathrm{g} h \mathrm{~h}}$ refers to the PS core bearing a PBMA shell of thickness, $h$. The red solid line denotes exponential decay excluding the data point for PS-PS $\left(\Delta T_{\mathrm{g}}=1\right)$. To display the actual $T_{\mathrm{g}}$ change, $T_{\mathrm{g}}(h)$ is shown in Figure S10 in the Supporting Information. The comparison with a flat PS/PBMA bilayer film is indicated by the red square symbols for the $T_{\mathrm{g}}(\mathrm{PBMA})$ in PBMA on the PS layer, whereas the blue triangle symbols are for $T_{\mathrm{g}}(\mathrm{PS})$ on the PBMA layer. ${ }^{26} h$ refers to the distance from the PS/PBMA interface.

(red circles vs red squares) but shows a similar trend. The representation of the $T_{\mathrm{g}}(h)$ dependence by an exponential decay (red solid line) cannot describe the initial $T_{\mathrm{g}}$ softening $(h<8 \mathrm{~nm})$ as indicated by the intercept $(h=0)$ value $(<1)$. Hence, the glass dynamics appears to reflect a topology effect since in bilayer films the layers are less confined along the direction parallel to the film. The higher $T_{\mathrm{g}}$ of PBMA even at about $60 \mathrm{~nm}$ thickness is apparent from the robust surface of PS-PBMA NPs compared to that of PBMA NPs with $T_{\mathrm{g}}=$ $293 \mathrm{~K}$.

The huge $T_{\mathrm{g}}$ enhancement effect in the PBMA shell suggests that free surface effects are significantly counter-balanced by the influence of the polymer-polymer interface. This is supported by the observation of very similar DSC $T_{\mathrm{g}}$ 's in the PS-PBMA NPs and the corresponding contiguous films of PS (cores) in the PBMA matrix (no free interface) in Figure S11. This effect mimics that observed in polymers tightly adsorbed on solid interfaces. Here, the effect of the free surface is erased by the presence of such an adsorbed layer. ${ }^{65}$ On the same line of reasoning, we can consider the (soft)polymer/(hard) polymer interface as analogous to that of polymer/hard substrates, where the analogy is marked by the presence of extensive van der Waals interaction involving several pinpoints on each polymer chain, whose effects propagate to the free interface. $^{60,65-68}$ However, it is worth noting that polymers in contact with an inorganic interface at best exhibit the bulk $T_{\mathrm{g}}$ or a $T_{\mathrm{g}}$ only a few degrees higher than that of the bulk. Thereby, the large $T_{\mathrm{g}}$ enhancement observed here may be at least partly explained by the presence of an interfacial mixed 
layer. The presence of such a layer has been demonstrated ${ }^{69}$ and was found to be about $8 \mathrm{~nm}$. This thickness may explain the very large $T_{\mathrm{g}}$ deviation found for PS-PBMA . The effect of the free (PBMA) surface is, however, evident in the NP's thermal behavior as indicated by the presence of the softening $T_{\mathrm{s}}\left(<T_{\mathrm{g}, 1}\right)$. It does relate to $T_{\mathrm{g}, 1}$ and the difference $T_{\mathrm{g}, 1}-T_{\mathrm{s}} \sim$ $15 \mathrm{~K}$ seems to be virtually independent of the PBMA thickness as shown in Figure 4a, suggesting a constant free surface contribution to $T_{s}$.

\section{CONCLUSIONS}

We synthesized core-shell nanoparticles with a PS core and low- $T_{\mathrm{g}}$ PBMA or high- $T_{\mathrm{g}}$ PMMA shell layer of varying thicknesses. We used Brillouin light scattering to measure the vibrational frequency spectrum of each nanoparticle type. We found that PS-PMMA core-shell particles exhibited a small, shell-thickness-independent increase in the elastic modulus. The elastic modulus of PS-PBMA core-shell particles decreased as the PBMA shell thickness increased. The change in the elastic modulus of the core-shell particles is not welldescribed by a finite-element analysis assuming bulk moduli for each component. Our results are more consistent with modulus hardening in the PMMA shell layer and softening in the PBMA shell layer under the spherical confinement induced by the NP core-shell geometry. Temperaturedependent Brillouin light spectroscopy reports the softening temperature as an increase in the vibrational frequency. Additionally, modulated DSC was used to measure the $T_{\mathrm{g}}$ of the nanoparticles. Both the softening temperature and $T_{\mathrm{g}}$ for PS-PMMA core-shell particles increased slightly, consistent with the $\sim 5 \mathrm{~K}$ higher PS-PMMA $T_{\mathrm{g}}$ compared with the PS $T_{\mathrm{g}}$. The softening temperature dramatically decreased for PSPBMA particles with thick shells. Modulated DSC measured two $T_{\mathrm{g}}$ 's for PS-PBMA NPs: a low shell-thickness-dependent $T_{\mathrm{g}}$ for the PBMA shell and a high, constant $T_{\mathrm{g}}$ for the PS core. The softening temperature measured via BLS was consistently $\sim 15 \mathrm{~K}$ below the low $T_{\mathrm{g}}$ measured by mDSC, suggesting that BLS is more sensitive to the shell of the nanoparticle and that the PBMA shell layer has enhanced mobility at the free surface. However, the PS-PBMA interaction dominates the mDSC $T_{\mathrm{g}}$ measured for the PBMA shell layer. Finally, according to a recently published article $^{28}$ on supported flat bilayer films, the $T_{\mathrm{g}}$ of PS with a rubbery topcoat is affected only for PS thicknesses below about $60 \mathrm{~nm}$. This is consistent with the unaffected $T_{\mathrm{g}}$ of the PS NPs $(d \sim 220 \mathrm{~nm})$ with a PBMA shell. In contrast, the $T_{\mathrm{g}}$ of PS on the top of an unsupported freePBMA film ${ }^{26}$ is unaffected only for PS thicknesses above 200 nm.

\section{ASSOCIATED CONTENT}

\section{S Supporting Information}

The Supporting Information is available free of charge on the ACS Publications website at DOI: 10.1021/acs.macromol.9b00766.

BLS spectra of all core-shell NPs (PS-PS, PS-PBMA, PS-PBMA 34 , PS-PBMA 8 , PS-PMMA , and PS$\mathrm{PMMA}_{16}$ ), relation of the interaction-induced mode, shear modulus data from characteristic particle vibration frequencies, $T_{\mathrm{g}}$ and $T_{\mathrm{s}}$ from BLS and $\mathrm{mDSC}$, comparison of experimental data with FEM, temperature dependency of eigenfrequencies of all core-shell NPs, heat maps of PS-PS and PS-PBMA groups, and $\mathrm{mDSC}$ thermograms (PDF)

\section{AUTHOR INFORMATION}

\section{Corresponding Author}

*E-mail: fytas@mpip-mainz.mpg.de.

ORCID $\odot$

Eunsoo Kang: 0000-0002-9894-0900

Bartlomiej Graczykowski: 0000-0003-4787-8622

Ulrich Jonas: 0000-0002-2161-4541

Daniele Cangialosi: 0000-0002-5782-7725

Rodney D. Priestley: 0000-0001-6765-2933

\section{Author Contributions}

This manuscript was written through contributions of all authors. All authors have given approval to the final version of the manuscript.

\section{Notes}

The authors declare no competing financial interest.

\section{ACKNOWLEDGMENTS}

E.K., B.G., and G.F. acknowledge the financial support by ERC Advanced Grant Smartphon (No. 694977). B.G. acknowledges the support from the First Team project (POIR.04.04.00-005D1B/18-00) granted by the Foundation for Polish Science. R.D.P. acknowledges the support of the National Science Foundation (NSF) Materials Research Science and Engineering Center Program through the Princeton Center for Complex Materials (DMR-1420541) and NSF CBET1706012. D.C. acknowledges financial support from the Basque Government (Grant No. IT-1175-19).

\section{REFERENCES}

(1) Karan, S.; Jiang, Z.; Livingston, A. G. Sub-10 Nm Polyamide Nanofilms with Ultrafast Solvent Transport for Molecular Separation. Science 2015, 348, 1347-1351.

(2) Jimenez-Solomon, M. F.; Song, Q.; Jelfs, K. E.; Munoz-Ibanez, M.; Livingston, A. G. Polymer Nanofilms with Enhanced Microporosity by Interfacial Polymerization. Nat. Mater. 2016, 15, 760767.

(3) Kumari, A.; Yadav, S. K.; Yadav, S. C. Biodegradable Polymeric Nanoparticles Based Drug Delivery Systems. Colloids Surf., B 2010, $75,1-18$.

(4) Moll, J. F.; Akcora, P.; Rungta, A.; Gong, S.; Colby, R. H.; Benicewicz, B. C.; Kumar, S. K. Mechanical Reinforcement in Polymer Melts Filled with Polymer Grafted Nanoparticles. Macromolecules 2011, 44, 7473-7477.

(5) Rogach, A.; Susha, A.; Caruso, F.; Sukhorukov, G.; Kornowski, A.; Kershaw, S.; Möhwald, H.; Eychmüller, A.; Weller, H. Nano- and Microengineering: 3-D Colloidal Photonic Crystals Prepared from Sub-Mm-Sized Polystyrene Latex Spheres Pre-Coated with Luminescent Polyelectrolyte/Nanocrystal Shells. Adv. Mater. 2000, 12, 333337.

(6) Still, T.; Cheng, W.; Retsch, M.; Sainidou, R.; Wang, J.; Jonas, U.; Stefanou, N.; Fytas, G. Simultaneous Occurrence of StructureDirected and Particle-Resonance-Induced Phononic Gaps in Colloidal Films. Phys. Rev. Lett. 2008, 100, No. 194301.

(7) Ediger, M. D.; Forrest, J. A. Dynamics near Free Surfaces and the Glass Transition in Thin Polymer Films: A View to the Future. Macromolecules 2014, 47, 471-478.

(8) McKenna, G. B.; Simon, S. L. 50th Anniversary Perspective: Challenges in the Dynamics and Kinetics of Glass-Forming Polymers. Macromolecules 2017, 50, 6333-6361. 
(9) Napolitano, S.; Glynos, E.; Tito, N. B. Glass Transition of Polymers in Bulk, Confined Geometries, and near Interfaces. Rep. Prog. Phys. 2017, 80, No. 036602.

(10) Bodiguel, H.; Fretigny, C. Reduced Viscosity in Thin Polymer Films. Phys. Rev. Lett. 2006, 97, 1-4.

(11) Yang, Z.; Fujii, Y.; Lee, F. K.; Lam, C.-H.; Tsui, O. K. C. Glass Transition Dynamics and Surface Layer Mobility in Unentangled Polystyrene Films. Science 2010, 328, 1676-1679.

(12) Chowdhury, M.; Guo, Y.; Wang, Y.; Merling, W. L.; Mangalara, J. H.; Simmons, D. S.; Priestley, R. D. Spatially Distributed Rheological Properties in Confined Polymers by Noncontact Shear. J. Phys. Chem. Lett. 2017, 8, 1229-1234.

(13) Chung, J. Y.; Nolte, A. J.; Stafford, C. M. Surface Wrinkling: A Versatile Platform for Measuring Thin-Film Properties. Adv. Mater. 2011, 23, 349-368.

(14) Zhai, M.; McKenna, G. B. Elastic Modulus and Surface Tension of a Polyurethane Rubber in Nanometer Thick Films. Polymer 2014, $55,2725-2733$.

(15) Keddie, J. L.; Jones, R. A. L.; Cory, R. A. Size-Dependent Depression of the Glass Transition Temperature in Polymer Films. Europhys. Lett. 1994, 27, 59-64.

(16) Ellison, C. J.; Torkelson, J. M. The Distribution of GlassTransition Temperatures in Nanoscopically Confined Glass Formers. Nat. Mater. 2003, 2, 695-700.

(17) Sharp, J. S.; Forrest, J. Free Surfaces Cause Reductions in the Glass Transition Temperature of Thin Polystyrene Films. Phys. Rev. Lett. 2003, 91, No. 235701.

(18) Tress, M.; Erber, M.; Mapesa, E. U.; Huth, H.; Müller, J.; Serghei, A.; Schick, C.; Eichhorn, K.-J.; Voit, B.; Kremer, F. Glassy Dynamics and Glass Transition in Nanometric Thin Layers of Polystyrene. Macromolecules 2010, 43, 9937-9944.

(19) Glynos, E.; Frieberg, B.; Oh, H.; Liu, M.; Gidley, D. W.; Green, P. F. Role of Molecular Architecture on the Vitrification of Polymer Thin Films. Phys. Rev. Lett. 2011, 106, No. 128301.

(20) Bäumchen, O.; McGraw, J. D.; Forrest, J. A.; Dalnoki-Veress, K. Reduced Glass Transition Temperatures in Thin Polymer Films: Surface Effect or Artifact? Phys. Rev. Lett. 2012, 109, No. 055701.

(21) Lan, T.; Torkelson, J. M. Methacrylate-Based Polymer Films Useful in Lithographic Applications Exhibit Different Glass Transition Temperature-Confinement Effects at High and Low Molecular Weight. Polymer 2014, 55, 1249-1258.

(22) Shamim, N.; Koh, Y. P.; Simon, S. L.; McKenna, G. B. Glass Transition Temperature of Thin Polycarbonate Films Measured by Flash Differential Scanning Calorimetry. J. Polym. Sci., Part B: Polym. Phys. 2014, 52, 1462-1468.

(23) Zhang, M.; Askar, S.; Torkelson, J. M.; Brinson, L. C. Stiffness Gradients in Glassy Polymer Model Nanocomposites: Comparisons of Quantitative Characterization by Fluorescence Spectroscopy and Atomic Force Microscopy. Macromolecules 2017, 50, 5447-5458.

(24) Roth, C. B.; McNerny, K. L.; Jager, W. F.; Torkelson, J. M. Eliminating the Enhanced Mobility at the Free Surface of Polystyrene: Fluorescence Studies of the Glass Transition Temperature in Thin Bilayer Films of Immiscible Polymers. Macromolecules 2007, 40, $2568-2574$.

(25) Roth, C. B.; Torkelson, J. M. Selectively Probing the Glass Transition Temperature in Multilayer Polymer Films: Equivalence of Block Copolymers and Multilayer Films of Different Homopolymers. Macromolecules 2007, 40, 3328-3336.

(26) Baglay, R. R.; Roth, C. B. Communication: Experimentally Determined Profile of Local Glass Transition Temperature across a Glassy-Rubbery Polymer Interface with a $\mathrm{T}$ g Difference of $80 \mathrm{~K}$. J. Chem. Phys. 2015, 143, No. 111101.

(27) Baglay, R. R.; Roth, C. B. Local Glass Transition Temperature $\mathrm{Tg}(\mathrm{z})$ of Polystyrene next to Different Polymers: Hard vs. Soft Confinement. J. Chem. Phys. 2017, 146, No. 203307.

(28) Chang, T.; Zhang, H.; Shen, X.; Hu, Z. Polymer-Polymer Interfacial Perturbation on the Glass Transition of Supported Low Molecular Weight Polystyrene Thin Films. ACS Macro Lett. 2019, 435-441.
(29) Christie, D.; Register, R. A.; Priestley, R. D. Direct Measurement of the Local Glass Transition in Self-Assembled Copolymers with Nanometer Resolution. ACS Cent. Sci. 2018, 4, 504-511.

(30) Christie, D.; Register, R. A.; Priestley, R. D. Role of Chain Connectivity across an Interface on the Dynamics of a Nanostructured Block Copolymer. Phys. Rev. Lett. 2018, 121, No. 247801.

(31) Tito, N. B.; Lipson, J. E. G.; Milner, S. T. Lattice Model of Mobility at Interfaces: Free Surfaces, Substrates, and Bilayers. Soft Matter 2013, 9, 9403.

(32) O'Connell, P. A.; McKenna, G. B. Rheological Measurements of the Thermoviscoelastic Response of Ultrathin Polymer Films. Science 2005, 307, 1760-1763.

(33) Stafford, C. M.; Harrison, C.; Beers, K. L.; Karim, A.; Amis, E. J.; VanLandingham, M. R.; Kim, H.-C.; Volksen, W.; Miller, R. D.; Simonyi, E. E. A Buckling-Based Metrology for Measuring the Elastic Moduli of Polymeric Thin Films. Nat. Mater. 2004, 3, 545-550.

(34) Rowland, H. D.; King, W. P.; Pethica, J. B.; Cross, G. L. W. Molecular Confinement Accelerates Deformation of Entangled Polymers during Squeeze Flow. Science 2008, 322, 720-724.

(35) Chung, J. Y.; Chastek, T. Q.; Fasolka, M. J.; Ro, H. W.; Stafford, C. M. Quantifying Residual Stress in Nanoscale Thin Polymer Films via Surface Wrinkling. ACS Nano 2009, 3, 844-852.

(36) Liu, Y.; Chen, Y. C.; Hutchens, S.; Lawrence, J.; Emrick, T.; Crosby, A. J. Directly Measuring the Complete Stress-Strain Response of Ultrathin Polymer Films. Macromolecules 2015, 48, 6534-6540.

(37) Tweedie, C. A.; Constantinides, G.; Lehman, K. E.; Brill, D. J.; Blackman, G. S.; Van Vliet, K. J. Enhanced Stiffness of Amorphous Polymer Surfaces under Confinement of Localized Contact Loads. Adv. Mater. 2007, 19, 2540-2546.

(38) Kang, E.; Kim, H.; Gray, L. A. G.; Christie, D.; Jonas, U.; Graczykowski, B.; Furst, E. M.; Priestley, R. D.; Fytas, G. Ultrathin Shell Layers Dramatically Influence Polymer Nanoparticle Surface Mobility. Macromolecules 2018, 51, 8522-8529.

(39) Sasaki, T.; Shimizu, A.; Mourey, T. H.; Thurau, C. T.; Ediger, M. D. Glass Transition of Small Polystyrene Spheres in Aqueous Suspensions. J. Chem. Phys. 2003, 119, 8730-8735.

(40) Zhang, C.; Guo, Y. L.; Priestley, R. D. Glass Transition Temperature of Polymer Nanoparticles under Soft and Hard Confinement. Macromolecules 2011, 44, 4001-4006.

(41) Feng, S.; Li, Z.; Liu, R.; Mai, B.; Wu, Q.; Liang, G.; Gao, H.; Zhu, F. Glass Transition of Polystyrene Nanospheres under Different Confined Environments in Aqueous Dispersions. Soft Matter 2013, 9, $4614-4620$.

(42) Zhang, C.; Boucher, V. M.; Cangialosi, D.; Priestley, R. D. Mobility and Glass Transition Temperature of Polymer Nanospheres. Polymer 2013, 54, 230-235.

(43) Perez-de-Eulate, N. G.; Di Lisio, V.; Cangialosi, D. Glass Transition and Molecular Dynamics in Polystyrene Nanospheres by Fast Scanning Calorimetry. ACS Macro Lett. 2017, 6, 859-863.

(44) Vogel, N.; de Viguerie, L.; Jonas, U.; Weiss, C. K.; Landfester, K. Wafer-Scale Fabrication of Ordered Binary Colloidal Monolayers with Adjustable Stoichiometries. Adv. Funct. Mater. 2011, 21, 30643073.

(45) Dimonie, V.; El-Aasser, M. S.; Klein, A.; Vanderhoff, J. W. Core-Shell Emulsion Copolymerization of Styrene and Acrylonitrile on Polystyrene Seed Particles. J. Polym. Sci., Polym. Chem. Ed. 1984, 22, 2197-2215.

(46) Hergeth, W.-D.; Bittrich, H.-J.; Eichhorn, F.; Schlenker, S.; Schmutzler, K.; Steinau, U.-J. Polymerizations in the Presence of Seeds: 5. Core-Shell Structure of Two-Stage Emulsion Polymers. Polymer 1989, 30, 1913-1917.

(47) Mattarelli, M.; Montagna, M.; Still, T.; Schneider, D.; Fytas, G. Vibration Spectroscopy of Weakly Interacting Mesoscopic Colloids. Soft Matter 2012, 8, 4235-4243.

(48) Guillet, Y.; Audoin, B.; Ferrié, M.; Ravaine, S. All-Optical Ultrafast Spectroscopy of a Single Nanoparticle-Substrate Contact. Phys. Rev. B 2012, 86, No. 035456. 
(49) Ayouch, A.; Dieudonné, X.; Vaudel, G.; Piombini, H.; Vallé, K.; Gusev, V.; Belleville, P.; Ruello, P. Elasticity of an Assembly of Disordered Nanoparticles Interacting via Either van Der WaalsBonded or Covalent-Bonded Coating Layers. ACS Nano 2012, 6, 10614-10621.

(50) Boechler, N.; Eliason, J. K.; Kumar, A.; Maznev, A. A.; Nelson, K. A.; Fang, N. Interaction of a Contact Resonance of Microspheres with Surface Acoustic Waves. Phys. Rev. Lett. 2013, 111, No. 036103.

(51) Girard, A.; Gehan, H.; Crut, A.; Mermet, A.; Saviot, L.; Margueritat, J. Mechanical Coupling in Gold Nanoparticles Supermolecules Revealed by Plasmon-Enhanced Ultralow Frequency Raman Spectroscopy. Nano Lett. 2016, 16, 3843-3849.

(52) Kim, H.; Cang, Y.; Kang, E.; Graczykowski, B.; Secchi, M.; Montagna, M.; Priestley, R. D.; Furst, E. M.; Fytas, G. Direct Observation of Polymer Surface Mobility via Nanoparticle Vibrations. Nat. Commun. 2018, 9, No. 2918.

(53) Xu, F.; Guillet, Y.; Ravaine, S.; Audoin, B. All-Optical in-Depth Detection of the Acoustic Wave Emitted by a Single Gold Nanorod. Phys. Rev. B 2018, 97, No. 165412.

(54) Johnson, K. L.; Kendall, K.; Roberts, A. D. Surface Energy and the Contact of Elastic Solids. Proc. R. Soc. A 1971, 324, 301-313.

(55) Lamb, H. On the Vibrations of an Elastic Sphere. Proc. London Math. Soc. 1881, s1-s13, 189-212.

(56) Cheng, W.; Sainidou, R.; Burgardt, P.; Stefanou, N.; Kiyanova, A.; Efremov, M.; Fytas, G.; Nealey, P. F. Elastic Properties and Glass Transition of Supported Polymer Thin Films. Macromolecules 2007, 40, 7283-7290.

(57) Torres, J. M.; Stafford, C. M.; Vogt, B. D. Manipulation of the Elastic Modulus of Polymers at the Nanoscale: Influence of UVOzone Cross-Linking and Plasticizer. ACS Nano 2010, 4, 5357-5365.

(58) Brune, P. F.; Blackman, G. S.; Diehl, T.; Meth, J. S.; Brill, D.; Tao, Y.; Thornton, J. Direct Measurement of Rubber Interphase Stiffness. Macromolecules 2016, 49, 4909-4922.

(59) Zhang, M.; Li, Y.; Kolluru, P. V.; Brinson, L. C. Determination of Mechanical Properties of Polymer Interphase Using Combined Atomic Force Microscope (AFM) Experiments and Finite Element Simulations. Macromolecules 2018, 51, 8229-8240.

(60) Song, J.; Kahraman, R.; Collinson, W. D.; Xia, W.; Catherine Brinson, L.; Keten, S. Temperature Effects on the Nanoindentation Characterization of Stiffness Gradients in Confined Polymers. Soft Matter 2019, 15, 359-370.

(61) Wingert, M. C.; Jiang, Z.; Chen, R.; Cai, S. Strong SizeDependent Stress Relaxation in Electrospun Polymer Nanofibers. J. Appl. Phys. 2017, 121, No. 015103.

(62) Richard-Lacroix, M.; Pellerin, C. Orientation and Partial Disentanglement in Individual Electrospun Fibers: Diameter Dependence and Correlation with Mechanical Properties. Macromolecules 2015, 48, 4511-4519.

(63) Midya, J.; Cang, Y.; Egorov, S. A.; Matyjaszewski, K.; Bockstaller, M. R.; Nikoubashman, A.; Fytas, G. Disentangling the Role of Chain Conformation on the Mechanics of Polymer Tethered Particle Materials. Nano Lett. 2019, 19, 2715-2722.

(64) Milenko, K.; Pissadakis, S.; Gkantzounis, G.; Aluculesei, A.; Fytas, G. Probing Stress-Induced Optical Birefringence of Glassy Polymers by Whispering Gallery Modes Light Localization. ACS Omega 2017, 2, 9127-9135.

(65) Perez-de-Eulate, N. G.; Sferrazza, M.; Cangialosi, D.; Napolitano, S. Irreversible Adsorption Erases the Free Surface Effect on the $\mathrm{Tg}$ of Supported Films of Poly(4-Tert-Butylstyrene). ACS Macro Lett. 2017, 6, 354-358.

(66) Burroughs, M. J.; Napolitano, S.; Cangialosi, D.; Priestley, R. D. Direct Measurement of Glass Transition Temperature in Exposed and Buried Adsorbed Polymer Nanolayers. Macromolecules 2016, 49, 4647-4655.

(67) Napolitano, S.; Wübbenhorst, M. The Lifetime of the Deviations from Bulk Behaviour in Polymers Confined at the Nanoscale. Nat. Commun. 2011, 2, No. 260.

(68) Napolitano, S.; Capponi, S.; Vanroy, B. Glassy Dynamics of Soft Matter under 1D Confinement: How Irreversible Adsorption
Affects Molecular Packing, Mobility Gradients and Orientational Polarization in Thin Films. Eur. Phys. J. E 2013, 36, 61.

(69) Siqueira, D. F.; Schubert, D. W.; Erb, V.; Stamm, M.; Amato, J. P. Interface Thickness of the Incompatible Polymer System PS/ PnBMA as Measured by Neutron Reflectometry and Ellipsometry. Colloid Polym. Sci. 1995, 273, 1041-1048. 